\title{
THE DIAGNOSIS OF APPENDICITIS.
}

\author{
By J. I. MUNRO BLACK, M.D., M.S., F.R.C.S. \\ (Hunterian Professor, Royal College of Surgeons; \\ From the Royal Victoria Infirmary, Newcastle upon Tyne.)
}

There has probably been as much written about appendicitis as any other single condition coming under the attention of the surgeon. Yet there is not yet by any means entire agreement upon all the many points connected with the management of these cases. I wish to confine myself at the moment to a consideration of the factors occurring under the heading of diagnosis. Of all diseases appendicitis is without doubt most suited to study and especially training of young men in surgery. Within the space of a short time one can examine the case, arrive at a diagnosis, operate to confirm that diagnosis and study the causal pathology at leisure just afterwards. What an opportunity, indeed, for demonstration of the actual pathology causing the patient's symptoms and a vindication or otherwise of the principles of diagnosis.

\section{PATHOLOGICAL FACTORS.}

Appendicitis has been divided into primary obstructive and primary infective, but in nearly all cases the precipitating factor is infection. A kink, a narrowing, or the presence of a stercolith, may be a facilitating feature, but the trouble is eventually started by a little infective swelling. Such a belief is really to satisfy the pathologists, from a clinical point of view the previous classification is highly recommended. The attack may begin with an obstruction to the appendicular lumen resulting in violent contractions of the appendix and possibly sympathetic increase of tone in the whole alimentary canal. This may overcome the obstruction in which case all returns to normal, or the process progresses until the appendix dies from interference with the blood supply perhaps coupled with infection when the muscular contractions cease, but infection spreads on. That is the so-called primary obstructive secondary infective appendicitis. In the other type the infection is the initial pathological process and does not give rise to obstruction, in some cases due to its incidence in the tip of the appendix. From such a beginning the infective process may be healed or may spread on to generalization but without obstructing the proximal lumen of the appendix. In some cases healing and fibrosis occurs which by possible proximal stricture formation may be a facilitating feature for a further attack. Such, very briefly, is the general type of pathology which is going to cause the symptoms of appendicitis.

\section{CORRELATION OF PATHOLOGY AND SYMPTOMATOLOGY.}

Next we must consider how the pathological processes cause the symptoms. It is accepted that the appendix itself is insensitive to all normal stimuli but that violent contractions of its musculature are perceptible as pain. These afferent stimuli are proceeding along the sympathetic nerves, whose peripheral ending is a nerve net and consequently impossible of localization to any one spot. Violent contraction of the intestine anywhere from about the duodenum to the transverse colon is felt as a colicky pain vaguely situated around the umbilicus. Thus if the appendix is contracting violently from any cause, obstruction or irritation, the symptom produced is colic in the mid-abdominal region. Although the contraction is local in its manifestation, the whole intestinal canal, bound into a harmonious unit by its nerve supply, seems to respond in sympathy. This vague increase of tone may result in one bowel movement, but later constipation; more interesting, 
however, is the way it explains the varied symptoms associated with the stomach. Vomiting has been given as one of the classical signs of appendicitis, but is by no means constant. If, as suggested, the stomach is merely hypertonic and probably hyperæsthetic, then if it contains anything it will empty itself, that is the patient will vomit; but if the stomach is empty then vomiting will not occur, but the patient experiences a feeling of nausea, akin to that noticed during the gastric hypertonia of hunger, the feeling of being " sick with hunger." Under such circumstances if anything is put into the stomach, usually some medicament to ease the colic, then the patient will probably vomit. These are the gastric symptoms found in actual practice to be associated with appendicular colic. It may be said in a general way that there are no other signs or symptoms associated with uncomplicated appendicular colic. Looked at from the other point of view, and this is the point of view we are dealing with, a patient is seen with vague abdominal colic accompanied by nausea or vomiting, what is the diagnosis? In the absence of any other clinical signs, examination of the abdomen revealing no abnormality, it can be said only that there is spasm or violent contraction of the intestine somewhere within the limits given above. The trouble may quite possibly lie in the appendix but there is no means of knowing at this stage. Supposing, however, that the source of the symptoms does lie in the appendix, then two things may happen: it may get better or it may get worse. If it gets better then the patient returns to normal, looking and feeling well and entirely normal to all physical examination. On the other hand, if it gets worse the musculature of the appendix for various reasons ceases to contract and in consequence the abdominal colic disappears. These symptoms, up to this stage, are dependent upon the healthy musculature of the appendix and are referred back through the afferent sympathetics, then imperfectly localized through consciousness. It must be realized that they are quite independent of the anatomical position of the appendix. Appendicular colic is situated vaguely around the umbilicus whether the appendix is in the right iliac fossa, in the pelvis, under the liver or even in the left iliac fossa. The associated characters of the pain are typical. It may be continuous or spasmodic, and makes the patient sweat and roll about: Usually the legs are drawn up and the abdomen compressed. Presumably in the obstructive cases, the stimulus to muscular contraction is increased tension in the walls of the appendix, and if the abdominal pressure is raised by straining and compression to equal the pressure inside the appendix then the tension in its walls will be removed and the colic eased.' When the colic is due to irritation no such possibility should arise. It is at this stage that localizing symptoms may develop depending upon the incidence of infection and the position of the appendix. These localizing symptoms and signs may be separated by some period of time from the foregoing, or may overlap them to some extent. In other cases, as will be discussed later, they do not occur at all

Already it has been stated that the appendix is insensitive to ordinary stimuli so that the inflamed organ is not tender or painful. The whole parietal peritoneum, however, is exquisitely sensitive to unusual stimuli and reacts as such to the products of inflammatory reaction. Although so sensitive, the pain is not felt actually in the peritoneum but projected or referred to the corresponding cutaneous nerve endings which in the case of the right iliac fossa are directly superimposed. In consequence, if the inflamed appendix is normally placed and in contact with the parietal peritoneum, the pain is felt directly over its situation, not necessarily at McBurney's point. Accompanying this pain is a reflex contraction of the local abdominal muscles, a protective phenomenon evidenced as rigidity. The localizing symptoms do then seem to depend upon the contact of 
an organically diseased appendix with the parietal peritoneum. If for some reason the appendix is shielded from the parietal peritoneum, perhaps lying behind the cæcum or wrapped in coils of small intestine, then localizing symptoms do not occur.

\section{DIAGNOSIS OF ACUTE APPENDICITIS.}

If these few simple principles are adopted it is surprising what a degree of ordered reason can be introduced into the diagnosis of appendicitis. With the appendix in its common position in the right iliac fossa lying in contact with the parietal peritoneum lining the anterior abdominal wall or reflected on to the posterior abdominal wall, an attack of appendicitis may produce the so-called classical symptoms and signs. Primary obstruction, or spasm of the appendix, cæcum or terminal ileum associated with irritation will give the mid-abdominal colic. Progress of the inflammation will result in cessation of the colic but cause a different type of pain in the right iliac fossa associated with rigidity. A patient suffering from colic rolls about, doubles up and compresses the abdomen; on the other hand when the pain is caused by the contact of a diseased appendix with the parietal peritoneum the pain is made worse by any movement, and if the patient lies still and holds his breath then there is usually no pain. So that as the pathology changes so do the symptoms and signs. The mid-abdominal colic is lost and is replaced by a different type of pain in another situation which is accompanied by local tenderness and rigidity. There are many cases, however, in which the spastic or obstructive element does not occur and the first indication is local pain, tenderness and rigidity over the appendix. This is not the classical picture of appendicitis but perfectly understandable when the pathology and the mechanism of the production of symptoms and signs is realized.

Supposing, now, that the appendix is lying behind the cæcum or otherwise shut off from contact with the parietal peritoneum. In the primary obstructive type the patient will experience the usual colic with no means for diagnosing its cause, which will, as the process progresses, disappear. One must now decide whether the cause of the colic has been removed, whether or not it lay in the appendix, in which case of course the patient is better, or whether the trouble lying in the appendix has progressed to destruction of the muscular mechanism and hence there is now a seriously inflamed or gangrenous appendix present. Odd little signs such as psoas rigidity or lumbar hyperæsthesia may help but very valuable is a leucocyte count, since a leucocytosis will indicate inflammation somewhere, and in the absence of other obvious causes will suggest appendicitis. Later the inflammatory process may spread until the reaction involves parietal peritoneum when local tenderness and rigidity will supervene; should this not occur it is possible that a partially painless lump, or abscess, will form. In primary. infective appendicitis, with the organ behind the cæcum, the primary colic may be missing and the trouble not declared until a fair amount of local damage is done. It is just such cases that progress painlessly to the formation of an abscess, the only previous evidence of trouble being that the patient is off colour or is bilious or has some vague intestinal disturbance. There may not be any justification for even a blood count. At operation, the surgeon is surprised to find a retro-cæcal gangrenous appendix and amazed that such serious pathology gave rise, to so little clinical trouble. It is not surprising really, since if a gangrenous appendix is entirely shielded from parietal peritoneum it will be undiagnosable unless by blind chance until it bursts its barriers or a lump is felt.

In the case of the pelvic appendix the story is just the same. There may or may not be, any early colic and nausea or vomiting. When the diseased 
appendix causes symptoms by its anatomical position they vary. It may lie in contact with some parietal peritoneum when perhaps suprapubic tenderness and rigidity will be evidenced, even pain referred down the thigh has been encountered. By fact of its proximity to the rectum, tenesmus or passage of mucous may occur and similarly the bladder by result of local irritation contracts at lower threshold resulting in frequency. The bladder wall may even be involved in the spread of the inflammatory process with exudation into its cavity resulting in albuminuria, pyuria or hæmaturia. In many such cases, however, maybe following an attack of abdominal colic which has passed off, the disease progresses until it is evidenced only by the production of a large pelvic abscess.

With the principles stated in one's mind it is found when dealing with cases of appendicitis that they do work out. Much of the teaching on the subject was in the past based on what have been termed typical cases, usually described as pain in the abdomen settling later in the right iliac fossa and accompanied by the various signs mentioned. To students and practitioners alike this has tended to suggest that if such signs and symptoms were not present then the case was not one of appendicitis, but it has been shown that this is by no means the case. In all cases a careful history should be taken and the possible causes of the trouble noted, being eliminated as necessary. An examination may show more probable causes or may not help, but in all cases an explanation of all symptoms and signs should be demanded. In many a diagnosis may be impossible, when the surgeon must acknowledge that fact and wait. It is urged that diagnosis is possible on grounds of reason, there is no indication for instance, of routine blood counts as an aid to diagnosis. In cases where the trouble is suggested by colic which has disappeared leaving no clinical signs, the blood count has real value. Even in what appear to be the most obvious cases a mistake may be made, since a suppurating lymph node in the mesentery of the terminal ileum may cause enteric spasm and colic, and by its contact with parietal peritoneum local tenderness and rigidity. A diagnosis of appendicitis would be perfectly justifiable, in fact one may even say that something must be wrong if it is not made. In the later stages of acute appendicitis the phenomenon of nervous fatigue make their appearances and local pain and tenderness are reduced, so that in a case of some standing, one is dependent very much on the history of a case for making a diagnosis.

\section{DIAGNOSIS OF CHRONIC APPENDICITIS.}

In the consideration of chronic appendicitis the same principles of diagnosis must be kept in mind. Actual chronic appendicitis, apart from specific infections is probably rare, and more common is a relapsing or recurrent condition either of an obstructive or infective nature or of course recurring attacks of actual appendicitis which for some reason avoid operation and recover. Recurring attacks of appendicular obstruction usually due to a kink or a band are evidenced as vague abdominal colic varying in severity. Usually at least one attack goes on to infection, localization is possible and the trouble is ended by operation. The obstruction may, however, be constantly present but never complete, in which case true chronic appendicular obstruction occurs, evidenced by slight vague pain or dyspepsia, perhaps diagnosed as biliousness or indigestion. In these obstructive cases clinical examination is quite negative as would be expected, but X-ray examination after a bismuth meal may help: the appendix may not fill or having filled may not empty, or again its mere shape when filled may suggest the possibility of obstruction. 
With chronic infective appendicitis the picture is different. There again recurring attacks may be evidenced. A history of attacks of local pain and tenderness may be caused over long periods by recurring apical appendicitis, each attack healing off a little more appendix until a completely fibrosed organ results, when natural cure has occurred. Similarly in a possible chronic infective condition a slight pain and tenderness may occur if the appendix is in contact with the parietal peritoneum, but as in the acute- cases a chronically inflamed appendix can be present without causing any local symptoms. At operation, however, it should be easy to decide whether the appendix has been the cause of the patient's symptoms. A stercolith in an apparently normal appendix or an organ which is merely kinked could not explain pain or tenderness in the side, and in the absence of colic or other lesser evidences of obstruction then the appendix has not been causing symptoms. It is conceivable, however, that a chronically inflamed appendix could cause vague abdominal symptoms and interference with general intestinal function, although not giving any local manifestations of its presence.

\section{CONCLUSION.}

With these general principles of abdominal diagnosis placed before us it is our duty to attempt an explanation of all symptoms by reason before operation, study of the living pathology at operation and of the morbid anatomy afterwards, and by this means teaching can be undertaken with confidence and the personal element largely eliminated. However brilliant a diagnostician any one person may be, unless he can give reasons for his deductions and by simple explanations to others enable them to approach easily his own excellence, then he is doing nothing to advance the science of diagnosis. 